\title{
A PROBABILISTIC METHOD FOR BLIND MULTI-USER DETECTION USING ARRAY OBSERVATIONS
}

\author{
Carles Antón-Haro \\ José A.R. Fonollosa \\ Javier R. Fonollosa \\ Dept. of Signal Theory and Communications - Universitat Politècnica de Catalunya.* \\ c/ Gran Capità s/n. Campus Nord UPC. 08034 Barcelona (SPAIN). \\ e-mail: $\{$ carles,adrian,fono\}@gps.tsc.upc.es
}

\begin{abstract}
In this paper, a blind algorithm for detecting active users in a DS-CDMA system is presented. This probabilistic algorithm relies on the theory of Hidden Markov Models (HMM) and is completely blind in the sense that no knowledge of the signature sequences, channel state information or training sequences is required for any user. Additionally, observation through an array of sensors is also considered. Performance is verified via computer simulations, showing the near-far resistance of the analyzed procedure.
\end{abstract}

\section{INTRODUCTION}

During the last two decades, multi-user detection in CDMA systems [1] has received great attention. In order to demodulate active users, two problems must be solved: channel distortion and nultiple access interference (MAI).

The use of antenna arrays can help in combating these problems. For example, in frequency non-selective channels, when there is no means of temporal diversity to overcome fading, an effective countermeasure is the use of antenna diversity.

Regarding MAI, whenever CDMA systems are strongly affected by the near-far problem, combining spread spectrum signals with adaptive array beamforming results in a more powerful system that rejects interferences and combats the multipath. Consequently, the performance and capacity of the system may be increased.

Besides, in recent years, a large effort has been made in developing blind detection algorithms. Such detection methods perform channel impulse response (CIR) acquisition and data detection without requiring additional information (in the form of users' signatures, timing or training sequences), thus adding flexibility to the system. In other words, blind receivers only make use of channel output together with limited statistical assumptions on the input signal. Blind equalization/estimation methods can be roughly classified in four families:

1. Bussgang algorithms,

2. Polyspectra and cumulant-based algorithms,

3. Cyclostationary statistics-based algorithms and

4. Probabilistic algorithms.

${ }^{*}$ This work was partially supported by the European Commission under ACTS, Project: ACO20 TSUNAMI (II), the National Plan of Spain, CICYT, TIC95-1022-C05-01, TIC96-0500-C10 01, the Generalitat de Catalunya, CIRIT, 1996SGR-00096. The TSUNAMI (II) consortium is formed by the following organizations: ERA Technology, Motorola ECID, Orange PCS, Robert Bosch, France Telecom CNET, CASA, University of Bristol, Aalborg University, Universitat Politècnica de Catalunya and Wireless Systems International.
Both Bussgang and Polyspectra methods are far from using all the known statistical information about the input signal, and algorithms based on cyclostationarity properties are limited to use second order statistics. On the contrary, probabilistic algorithms [2]-[5], which lead to joint channel estimation and data detection often on a basis of a Maximum Likelihood (ML) criterion, take advantage of this information more efficiently. These methods exhibit higher computational complexity and, unlike algorithms belonging to the second and third groups, may suffer from convergence to spurious solutions. However, probabilistic algorithms clearly outperform in other fields. For example, in comparison with Bussgang and Polyspectra techniques, more accurate channel identification can be achieved from a reduced number of samples.

The approach considered in this paper consists in a probabilistic algorithm on the basis of the Baum\&Welch (BW) reestimation procedure. This BW-based algorithm, which relies on the theory of Hidden Markov Models (HMM) $[6,7]$, was initially proposed in a single-user case in [8], further developed in [9] and applied to perform jointly blind channel estimation and sequence detection in a CDMA environment in [3]. In this paper, the algorithm is extended to a multisensor case.

The technique we are proposing is completely blind in the sense that no knowledge of signature waveforms, timing, amplitudes or training sequences is required for any user. Moreover, an estimate of each user's signature convolved with the physical channel response is adaptively obtained; estimated data sequences are also provided.

In $[4,5]$, a closely-related technique can be found. In that case, the authors employ a Viterbi-based algorithm to perform blind channel identification. A detailed comparison between the Viterbi and Baum\&Welch-based approaches is carried out in [3].

\section{SYSTEM MODEL}

We consider the general asynchronous multiple-access channel model. The multichannel receiver employs a $P$ sensor array in which the signal received by sensor $p, p=1, \ldots, P$ is given, regardless of the array structure by,

$$
r_{p}(t)=\sum_{n} \sum_{k=1}^{K} b_{k}[n] h_{k p}(t-n T, t)+\sigma w_{p}(t)
$$

where $h_{k p}(t-n T, t)$ is the overall complex channel impulse response of user $k$ at sensor $p$, given by the convolution of its signature sequence, physical channel and the receiving filter impulse responses. It incorporates the amplitude and the delay for user $k$ at sensor $p$, and its duration is assumed to be smaller or equal to $L$ symbol periods. The total number of active users is $K$ and their transmitted data sequences are binary independent symbols $b_{k}[n] \in\{1,-1\}$. The 
symbol rate is $1 / T$ and $w_{p}(t)$ is normalized temporal and spatially white Gaussian noise. The multi-sensor multipleaccess channel is sampled at a rate $f_{s}=1 / T_{s}=M / T$ to derive the discrete vector sequence $\mathbf{r}[n]$

$$
\mathbf{r}[n]=\left[\mathbf{r}_{1}[n]^{T}, \ldots, \mathbf{r}_{P}[n]^{T}\right]^{T}
$$

where the $M$ length vector corresponding to a $p^{\text {th }}$ sensor is given by

$$
\mathbf{r}_{p}[n]=\left[r_{p}(n T), \ldots, r_{p}\left(n T+(M-1) T_{s}\right)\right]^{T} .
$$

The observation $\mathbf{r}[n]$ is modeled as a $M P$-length vector, probabilistic function of a state vector $s[n]$,

$$
\mathbf{r}[n]=\mathbf{H}[n] \mathbf{s}[n]+\mathbf{w}[n] .
$$

Since, at any given time, a maximum of $L$ symbols for each user affect the observation, there are $N=2^{K L}$ possible state vectors corresponding to all combinations of $L$ binary symbols of the $K$ active users. Note that the number of state vectors is independent of the array size and therefore the number of sensors does not increase the complexity of the algorithm. We denote each of the possible states as the $K L$ length vectors $\mathrm{s}_{j}$,

$$
\mathbf{s}_{j} \in \mathcal{S}=\left\{\mathbf{s}_{1}, \mathbf{s}_{2}, \ldots, \mathbf{s}_{N}\right\}
$$

such that

$$
\begin{aligned}
\mathbf{s}_{j} & =\left[\mathbf{s}_{j 1}^{T}, \ldots, \mathbf{s}_{j K}^{T}\right]^{T}, \\
\mathbf{s}_{j k} & =\left[s_{j k}^{(0)}, \ldots, s_{j k}^{(L-1)}\right]^{T}, s_{j k}^{(l)} \in\{1,-1\} .
\end{aligned}
$$

The state at time instant $n T$ is denoted by $s[n] \in \mathcal{S}$. The $(M P \times K L)$ matrix $\mathbf{H}[n]$ depends of the overall discrete impulse response for each user, denoted by the matrix $\mathbf{H}_{k}[n]$,

$$
\mathbf{H}[n]=\left[\mathbf{H}_{1}[n], \ldots, \mathbf{H}_{K}[n]\right] .
$$

Each of these matrices incorporates a vector response for each of the $L$ symbols that may be present in the observation due to the ISI,

$$
\mathbf{H}_{k}[n]=\left[\mathbf{h}_{k 0}[n], \ldots, \mathbf{h}_{k(L-1)}[n]\right]
$$

which again includes the vector response for each of the $P$ sensors,

$$
\mathbf{h}_{k l}=\left[\mathbf{h}_{k l l}[n]^{T}, \ldots, \mathbf{h}_{k l P}[n]^{T}\right]^{T}
$$

and finally the resulting signature for each user, symbol and sensor

$$
\mathbf{h}_{k l p}[n]=\left[\begin{array}{c}
h_{k p}((n+l) T, n T) \\
\vdots \\
h_{k p}\left((n+l) T+(M-1) T_{s}, n T\right)
\end{array}\right] .
$$

The noise is modeled as the $M P$ length vector

$$
\mathbf{w}[n]=\left[\mathbf{w}_{1}[n]^{T}, \ldots, \mathbf{w}_{P}[n]^{T}\right]^{T}
$$

that includes the observed noise for each sensor at the sample rate,

$$
\mathbf{w}_{p}[n]=\sigma\left[w_{p}(n T), \ldots, w_{p}\left(n T+(M-1) T_{s}\right)\right]^{T} .
$$

\section{THE MULTI-USER ADAPTIVE BAUM\&WELCH (MABW) ALGORITHM}

The BW algorithm is essentially identical to the EM method and it is known to lead, at least, to a local maximum of the likelihood function [7]. When dealing with timevarying channels, an adaptive version called ABW (Adaptive Baum\&Welch) can be considered [8].

To achieve channel identification, the BW-based algorithm operates on a trellis-like structure having, in a multiuser case, $N=2^{K L}$ states. First, the probability, $\gamma_{j}[n]$, of being in state $j: 1 . . N$ in the trellis at time instant $n$ given the sequence and the HMM is computed by means of the Forward-Backward algorithm [6]. Second, the estimated multi-user CIR is updated at the symbol rate considering the steepest-descent adaptation scheme:

$$
\hat{\mathbf{H}}[n]=\hat{\mathbf{H}}[n-1]+\mu_{h} E\left[\mathbf{e}[n] \mathbf{s}[n]^{H}\right],
$$

where

$$
\mathrm{e}[n]=\mathbf{r}[n]-\mathrm{m}[n]=\mathbf{r}[n]-\hat{\mathbf{H}}[n] \mathbf{s}[n]
$$

and $\mu_{h}$ is the adaptation constant. Note that such CIR is, accordingly to the signal model, the convolution of the signatures with the physical channel response. In our blind environment, the expectation in the gradient term will be computed on the basis of the received sequence up to instant $n+\Delta$ (where the lag $\Delta$ is a design parameter taking typical values of $4-8$ symbols) and the model at instant $n-1$ :

$$
\begin{aligned}
\hat{\mathbf{H}}[n] & =\hat{\mathbf{H}}[n-1]+\mu_{h} E_{r_{n+\Delta, \lambda_{n-1}}}\left[\mathrm{e}[n] \mathbf{s}[n]^{H}\right] \\
& =\hat{\mathbf{H}}[n-1]+\mu_{h} \sum_{j=1}^{N} \gamma_{j}[n] \mathrm{e}_{j}[n] \mathbf{s}_{j}^{H}
\end{aligned}
$$

and

$$
\mathbf{e}_{j}[n]=\mathbf{r}[n]-\mathbf{m}_{j}[n]=\mathbf{r}[n]-\hat{\mathbf{H}}[n-1] \mathbf{s}_{j} \quad j=1 . . N .
$$

Data detection is performed together with the adaptation following an individually most-likely state criterion [6].

\subsection{Overcoming Local Maxima}

The MABW adaptation algorithm might suffer from convergence to local maxima yielding incorrect estimates of the CIRs. Those maxima appear as a consequence of two fairly independent phenomena [5]: severe ISI due to multipath propagation and the multi-user structure of the received signal provided it is affected by the near-far effect. The first circumstance rarely occurs in mobile channels with low to moderate ISI. Therefore, we will focus on the mechanism to overcome the second phenomenon.

When a multi-user signal is affected by the near-far effect, the algorithm tends to split the CIR estimate of the user(s) of greatest magnitude among the weakest(s) ones since this solution locally maximizes the likelihood function. Nevertheless, this extent can be easily detected since, in those cases, estimated data sequences for the involved users are identical (up to a sign change). The strategy to overcome this problem consists in checking periodically for this total coincidence (coherence checking) and, if necessary, rearranging the overall CIR estimates obtained so far (coherence compensation) [5].

\subsection{Convergence study}

\section{SIMULATION RESULTS}

Although in DS-CDMA systems user separation mainly relies on the orthogonality among spreading sequences, when multi-sensor observation is employed a direction of arrival (DoA) criterion can be considered as well. The aim of this section is to analyze the feasibility of including such spatial 
information, to be acquired blindly, in order to distinguish users.

The system under study operates at the symbol rate employing a BPSK modulation scheme. In all cases, $K=4$ users impinging on a linear array with $P=7$ sensors $(\lambda / 2$ spaced) contributed to the CDMA signal. For simplicity, users are assumed to be synchronous. Gold sequences with

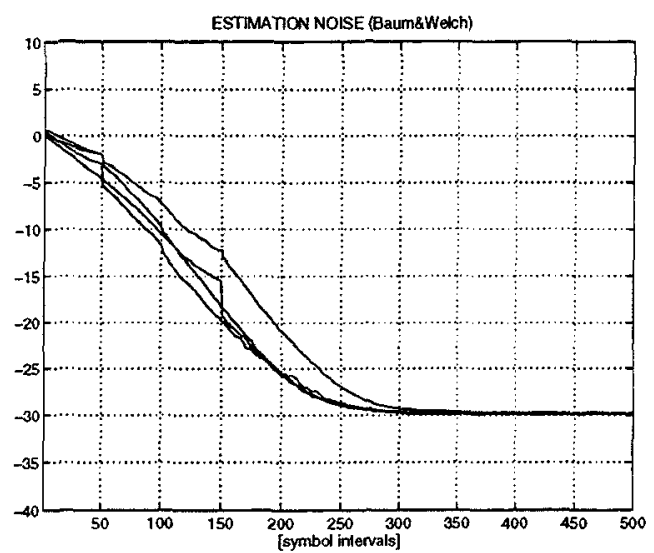

Figure 1. Learning curves with signatures. DoA: $\mathbf{9 0 , 9 0 , 9 0 , 9 0 . ~}$

$M=31$ chips were used as spreading sequences, that is, $T_{c}=T / 31$, being $T$ the symbol time; coherence checkings were performed every $S=50$ symbols. A stationary and single-path model was considered for the channel. Simulation results are averaged over 50-run tests and the performance measure is the CIR estimation noise defined (for each user) as:

$$
\xi_{k}[n]=\frac{1}{M L P} \sum_{l=0}^{L-1} \sum_{p=0}^{P-1}\left\|\mathbf{h}_{k l p}(n)-\hat{\mathbf{h}}_{k l p}(n)\right\|^{2}
$$

In Fig. 1, the DoA for all users is 90 degrees and the SNR, for all users as well, equals to $10 \mathrm{~dB}$. In this case, the only criterion to separate users is the orthogonality among spreading sequences. It can be observed that the steady state is reached around symbol $n=300$.

Assuming plane-wave propagation, the set of relative phase-delays observed at the different sensors (i.e. steering vectors) can be viewed as a kind of spatial signature. Furthermore, if the directions of arrival are apart enough, no spreading sequence might be required to distinguish users.

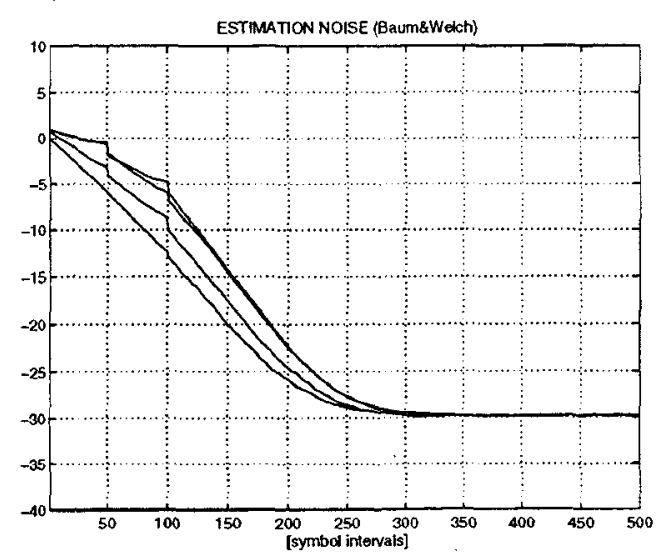

Figure 2. Learning curves without signatures. DoA: $90,55,75,105$.

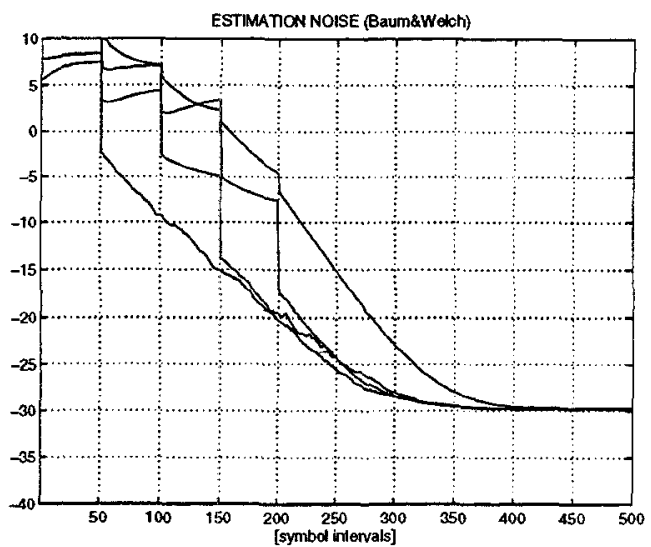

Figure 3. Learning curves with signatures and nearfar effect. DoA: 90,55,75,105.

This extent is reflected in Fig.2. The directions of arrival were, respectively, of $90,55,75$ and 105 degrees from broadside reverting in a maximum value for the normalized crosscorrelation between steering vectors of 0.1053 . Despite of the fact that no user-specific signature is considered, the algorithm is capable of separating active users. This fact can be taken into account in order to improve system capacity by employing space-division multiple-access (SDMA), that is, assigning the same spreading sequences to users with large angular separation. Furthermore, no additional improvement seems to be obtained by using spreading sequences when signals impinge from very different directions (see Fig.3)

It should be pointed out that sudden jumps in the curves are caused by the mechanism proposed to overcome local maxima (coherence compensations) which suddenly rearranges current CIR estimates. Also note that, regardless of the simplicity in the channel model, (i.e. plane-wave propagation, direct line-of-sight, no multipath propagation) no assumption concerning to the propagation conditions is made within the MABW algorithm. However, the algorithm takes advantage from those ideal conditions. That lack of assumptions adds flexibility to the algorithm which can be employed unchanged in a wide variety of propagation environments.

In Fig. 3 users are received with dissimilar levels $(\mathrm{SNR}=25,18,10,10 \mathrm{~dB})$ whereas the rest of parameters remain unchanged with respect to Fig.1. A near-far resistant behaviour is clearly observed. Now, strongest users are detected more rapidly since they can be easily distinguished from the weakest ones.

\subsection{Tracking properties}

For this section, we propose the following scenario: three users propagating through sensor-independent Rayleighfading channels and being received by a linear array. The averaged SNR at the input of each sensor is: 25,15 and 10 $\mathrm{dB}$ for the first, second and third users respectively. Propagation delays, relative to the first user, are $\tau_{1}=0, \tau_{2}=1 T_{c}$ and $\tau_{3}=2 T_{c}$, where $T_{c}$ is the chip time. The spreading sequences are Gold signals of length $M=7$. The mobile's speed is $200 \mathrm{~km} / \mathrm{h}$ and the carrier frequency $1800 \mathrm{MHz}$ thus generating a maximum Doppler frequency shift of $330 \mathrm{~Hz}$. The duration of the symbol (bit time) is $3.69 \mu \mathrm{s}$. Recall that the CIR estimated at each sensor and for each user is, in fact, the convolution of the physical channel response observed through that sensor with the user's signature. Note also that, in this case, the physical channel response is just a time-varying complex multiplicative factor, that is, flat fading is assumed. 

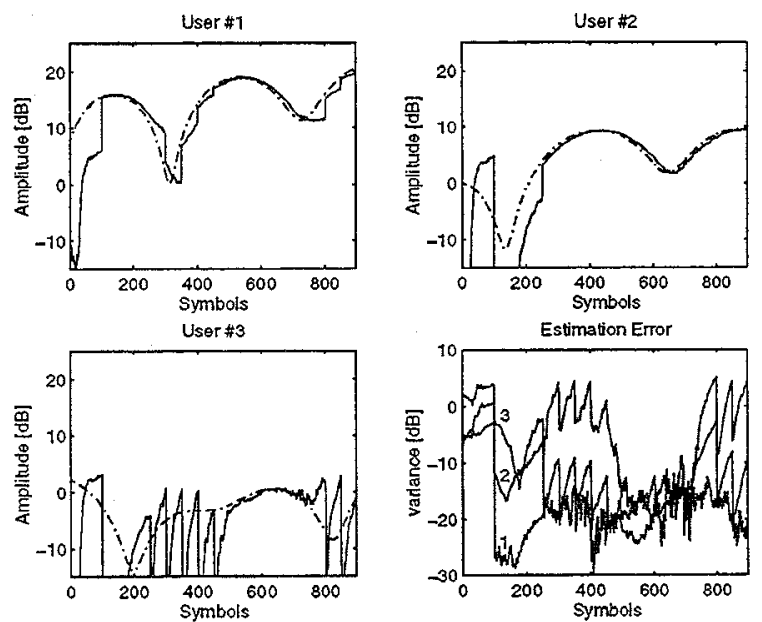

Figure 4. Performance of the MABW algorithm without array observations
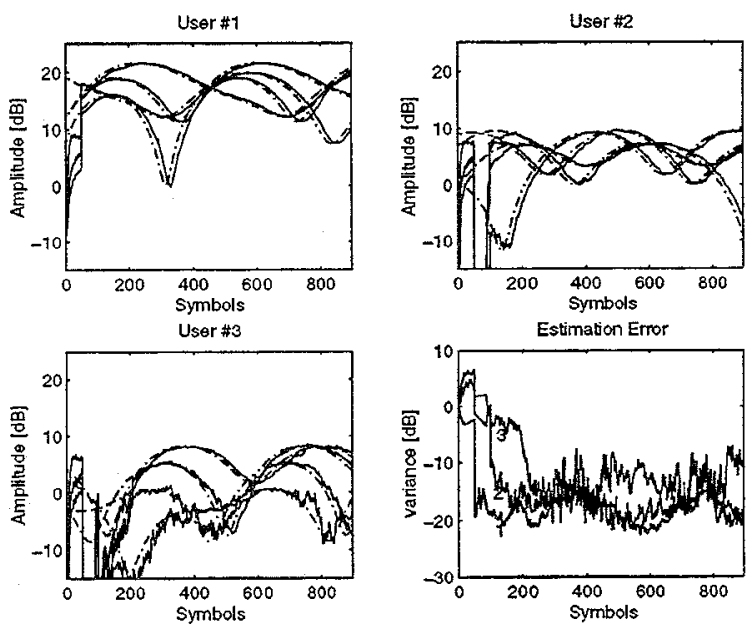

Figure 5. Performance of the MABW algorithm with array observations

In Figure 4, the multi-user received signal is observed through a single sensor. Subplot 1 is dedicated to show the evolution of the estimate of such multiplicative factor (in amplitude and averaging for all chips in a bit period) for user \#1 (dashed line: actual value). Subplots 2 and 3 correspond to the rest of users. We observe that within the first 200 symbols, strongest user's CIR splits among different user's CIR estimates. After coherence compensation at time instant $n=100$ tracking for the furst user starts whereas the rest of CIRs estimates are reset to their initial estimates. From that moment on, the estimates for such users should start improving until tracking. However, because of the fade experienced by user \#3, no tracking is attained for this user until time instant $n=500$. Actually, within symbols 200 to 500 when user \#3 suddenly disappears, CIR estimate for user \#1 splits again between those two users causing continuous coherence compensations. This happens again at the end of the burst (symbol $n=700 \mathrm{ap}$ proximately). Therefore, not only the estimate of the user is degraded, but also that of another user. This extent can be observed in subplot 4 where the evolution of the MSE in the estimation of the signatures (normalized by the received power) for all active users is shown. This behaviour should be also taken into account at the time of estima- ting the number of active users; if overestimated, strongest users' CIR estimates will split among several empty CIR estimates causing severe degradation in performance.

Figure 5 is devoted to show the behaviour of the MABW algorithm when deploying a 4-sensor array. Since no spatial information is embedded in the sensor-independent Rayleigh-fading channels, improvements in performance will only rely, unlike in the previous subsection, in the diversity introduced by the array observations. Note that signal observed through the first sensor corresponds to the same realization as in the single-sensor case. As before, subplots 1 to 3 are dedicated to show the evolution of the estimate of such multiplicative factor (in amplitude) for user \#1 to \#3 as observed through sensors 1 to 4 . Now, an accurate fading-resistant tracking of the amplitude is observed for all active users. Also, signature estimates are obtained within the first 200 symbols and the variance of the estimation error keeps at safe levels (less than $-10 \mathrm{~dB}$ ) throughout the whole burst. Note that the performance of the algorithm is satisfactory despite of the high near-far ratio $(15 \mathrm{~dB})$.

\section{CONCLUSIONS}

In this paper, a blind algorithm for multi-user detection has been extended to include array observation. First, a brief overview of the MABW algorithm has been given making a point of a mechanism to avoid ill-convergence when the incoming signal is affected by the near-far effect. Second, advantages arising from the use of a multi-sensor framework in terms of user distinction on a DoA basis (SDMA) have been analized. Finally, an example showing the improved resistance to deep fades of the algorithm when using array observations has been provided.

\section{REFERENCES}

[1] S. Verdú, "Minimum probability of error for asynchronous gaussian multiple-access channels," IEEE Trans. on Information Theory, vol. IT-32, pp. 85-96, Jan. 1986

[2] M. Ghosh and C.L. Weber, "Maximum likelihood blind equalization," Proc. SPIE Adaptive Signal Processing, vol. 1565 , pp. $188-195,1991$.

[3] C. Antón-Haro, J.A.R. Fonollosa, Z. Zvonar, and J.R. Fonollosa, "Probabilistic algorithms for blind adaptive multiuser detection," Submitted to IEEE Trans. on Signal Processing, 1996.

[4] N. Seshadri, "Joint data and channel estimation using fast blind trellis search techniques," IEEE Trans. on Communications, vol. COM-42, pp. 1000-1011, Feb/March/April 1994.

[5] J.R. Fonollosa, J.A.R. Fonollosa, Z. Zvonar, and J. Vi$\mathrm{dal}$ "Blind multiuser identification and detection in CDMA systems," in Proc. IEEE Int. Conf. Acoust. Speech and Signal Processing, (Detroit (USA)), pp. 18761879, May 1995.

[6] L. Rabiner, "A tutorial on hidden markov models and selected applications in speech recognition," Proc. of the IEEE, vol. 77 , pp. 257-286, Feb. 1989.

[7] L.E. Baum, T. Petrie, G. Soules, and N. Weiss, "A maximization technique ocurring in the statistical analysis of probabilistic functions of markov chains," The Annals of Mathematical Statistics, vol. 41, no. 1, pp. 164-171, 1970 .

[8] J.A.R. Fonollosa and J. Vidal, "Application of hidden markov models to blind channel characterization and data detection," in Proc. IEEE Int. Conf. Acoust. Speech and Signal Processing, (Australia), pp. 185-188, April 1994.

[9] C. Antón-Haro, J.A.R. Fonollosa, and J.R. Fonollosa, "Blind channel estimation and data detection using hidden markov models," To appear in IEEE Trans. on Signal Processing-Special Issue on Signal Processing for Advanced Communications, Jan. 1997. 